Network Working Group

Request for Comments \#152

NIC \#6756

Category: G.3

Obsoletes: None

Updates: None

Response to RFC \#116
M. Wilber

10 May 71

SRAI

\title{
SRI ARTIFICIAL INTELLIGENCE STATUS REPORT
}

The Stanford Research Institute's Artificial Intelligence Group (SRAI in the four-letter abbreviations) expects connection to the ARPA net as a research center after conversion this summer to a TENEX from our current PDP-10. Our connection will be through the IMP already at SRI for the Network Information Center and through a PDP-15 serving our PDP-10 as a controller of peripherals. Our hardware interface to the IMP is currently in the bidding process, and we intend to use as much as possible of the TENEX network software. (Probably all we will need to change is the lowest level, most strongly hardware-oriented part of the NCP.)

The most optimistic estimate we can give for functional connection to the network is mid-July 1971. We are currently devoting the energies of our system support group to the accomodation of various hardware and design changes, and so our contact with the Network Working Group has been only minimal and passive. It is entirely conceivable that we may find our participation tending to strength and activeness as we cross our other bridges.

We can project our participation in the network into the first few months of our connection. We can support several simultaneous outside users on a system nominally up during the business day and often up at other times. Lapses in continuity of system operation are usually due to scheduled maintenance or hardware failures and tend to occur at intervals of either an hour or several days with remarkable consistency. The principal service we offer to other network participants is the availability of various parts of our own research software. The most notable examples are QA3.6, a first-order resolution theorem prover; STRIPS, an extralogical problem solver; and possibly QA4, a language oriented toward problem-solving strategies. The services we can anticipate requesting of the network are of two kinds: We could conceivably use other people's artificial intelligence programs on a trial basis; and we might use the network to make occasional contact with other people in the network.

[ This RFC was put into machine readable form for entry ]

[ into the online RFC archives by BBN Corp. under the ]

[ direction of Alex McKenzie. 12/96 ] 